\title{
Acycloguanosine: antiviral activity in the rabbit cornea
}

\author{
M. G. FALCON AND B. R. JONES \\ From the Department of Clinical Ophthalmology, Institute of Ophthalmology, University of London
}

SUMMARY We describe experiments, using the multiple microinoculation technique, to produce superficial herpes simplex keratitis in the rabbit cornea, which showed a potent antiviral effect of acycloguanosine.

Although they are usually effective for the treatment of epithelial herpes simplex keratitis, idoxuridine (IDU), adenine arabinoside (ara-A), and trifluorothymidine $\left(\mathrm{F}_{3} \mathrm{~T}\right)$ have limitations of solubilities and toxicity which render them inadequate for the management of deep herpetic disease. Acycloguanosine (Wellcome 248U) shows promise of overcoming these limitations. It is soluble in water (as the sodium salt), and has low toxicities from both topical ocular and systemic administration in several animal species (Schaeffer et al., 1978; Tucker et al., 1978) and man. It has potent antiviral activity against herpes simplex virus (HSV) types I and II, both in vitro and in vivo (Elion et al., 1977; Bauer et al., 1979).

As a preliminary to our first clinical trial of $248 \mathrm{U}$, which has now been completed (Jones et al., 1979), and in order to compare data from our own laboratory with those of others (Bauer et al., 1979) we investigated the efficacy and tolerability of $248 \mathrm{U}$ in our rabbit corneal lesion inhibition system (Falcon and Jones, 1977a).

\section{Methods}

Details of the multiple microinoculation technique of Jones and Al-Hussaini, and of its modification to produce a corneal epithelial lesion inhibition assay, are published elsewhere (Jones and Al-Hussaini, 1963; Falcon and Jones, 1977a). In summary, 25 circular sites are inoculated on each eye of anaesthetised adult Dutch rabbits by means of a capillary tube of $1 \mathrm{~mm}$ diameter filled with $\mathrm{pH} 8$ strain of HSV (of $1.5 \times 10^{6}$ plaque-forming units $/ \mathrm{ml}$ ). Treatment generally begins 2 hours after inoculation and is administered 5 times daily. 48 hours after inoculation each site is scored 0 to 4 according to

Address for reprints: M. G. Falcon, FRCS, Department of Clinical Ophthalmology, Moorfields Eye Hospital, City Road, London EC1V 2PD

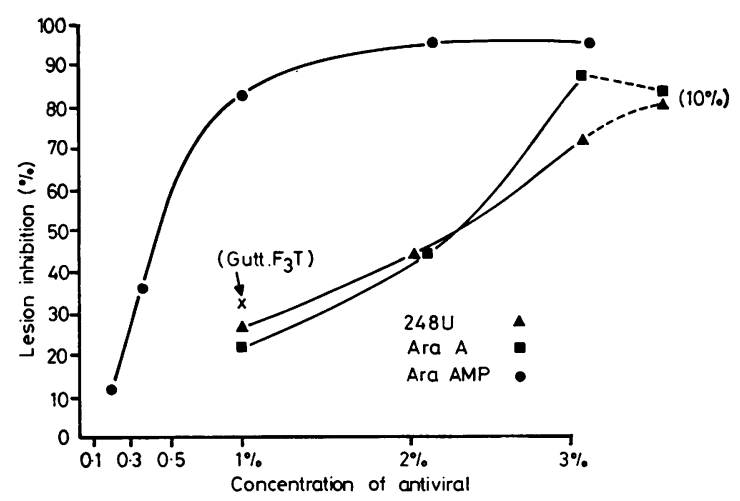

Fig. 1 Dose/response curves for 248U, ara- $A$ and ara-AMP ointments. Treatment was given 5 times daily, starting 2 hours after inoculation. Eyes scored 48 hours after inoculation

the number of sites infected, and a corneal epithelial lesion inhibition assay (CELIA) is produced by comparing treated with control eyes in groups of 3 or 4 rabbits (which gives statistically valid results). The advantage of this lesion-inhibition system is that the lesions are small and discrete at the time of scoring, which provides rapid, accurate, and repeatable results from the minimum of animals, and the experiment can be terminated before the eyes have become uncomfortable.

\section{Results}

Experiments were performed to give a dose/response curve for $248 \mathrm{U}$ ointment. This was compared with ara-A ointment, and ara-A $5^{\prime}$ monophosphate (ara-AMP) ointment, which had been shown previously to give greater lesion inhibition than any other antiviral tested in this system (Falcon and Jones, 1977b). It was also compared with standard $0.5 \%$ IDU ointment and with $1 \% \mathrm{~F}_{3} \mathrm{~T}$ drops (Fig. 1 ). We had shown previously that a single dose of 
ara-AMP given directly after inoculation had an almost catastrophic lesion-inhibiting effect, whereas IDU, ara-A, or $F_{3} T$ had little effect (Falcon and Jones, 1977b). 248U was employed in the same system and likewise had very little effect (Fig. 2).

In experiments to investigate the effect of systemic $248 \mathrm{U}, 50 \mathrm{mg} / \mathrm{kg}$ of $248 \mathrm{U}$ was given intravenously twice daily, the injections starting 2 hours after inoculation, and this experiment was continued for 72 hours. At 48 hours there was a small difference in scores between treated and control animals, and this had increased very considerably by 72 hours (Fig. 3).

Although this model, and particularly the scoring system, were derived primarily for lesion-inhibition experiments, we attempted to use them to assess therapy of established lesions: treatment began 48 hours after inoculation and was given 5 times daily. Scoring was carried out immediately before the beginning of the treatment and subsequently after 3 and 5 more days. We compared control, oculentum IDU $0.5 \%$, oculentum ara-A $1 \%$, oculentum araAMP $1 \%$, guttae ara-AMP $1 \%$, guttae $F_{3} T 1 \%$, and guttae $248 \mathrm{U} 1 \%$. It proved impossible to produce meaningful scores, since the lesions 48

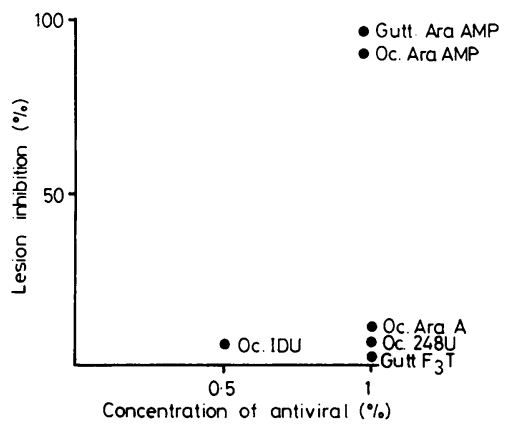

Fig. 2 Lesion inhibition from single doses of various antivirals. Treatment was given once immediately after inoculation, and the eyes were scored 48 hours later

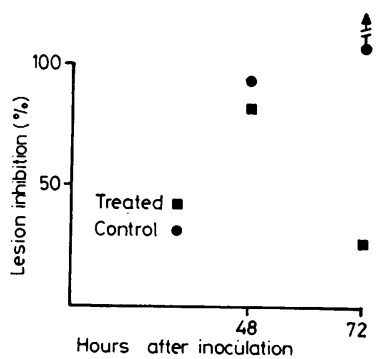

Fig. 3 Lesion inhibition 48 and 72 hours after inoculation: $50 \mathrm{mg} / \mathrm{kg}$ of $248 \mathrm{U}$ was given intravenously twice daily, starting 2 hours after inoculation
Table 1 Number of eyes healed (out of 3 in each group), 3 and 5 days after start of antiviral treatment, which was given 5 times daily, starting 48 hours after inoculation

\begin{tabular}{lll}
\hline \multirow{2}{*}{ Antiviral } & \multicolumn{2}{l}{ Number healed } \\
\cline { 2 - 3 } & At 3 days & At 5 days \\
\hline Control & 0 & 0 \\
Oculentum IDU $0.5 \%$ & 0 & 0 \\
Oculentum Ara A $1 \%$ & 0 & 0 \\
Oculentum Ara AMP $1 \%$ & 0 & $0 *$ \\
Guttae $\mathrm{F}_{3} \mathrm{~T} 1 \%$ & 2 & 3 \\
Guttae $248 \mathrm{U} 1 \%$ & 3 & 3 \\
\hline
\end{tabular}

* Severe toxic epitheliopathy at this stage

hours from inoculation had already spread away from the inoculation sites. We therefore merely scored each eye as 'healed' or 'unhealed' (Table 1).

\section{Discussion}

Fig. 1 shows that the dose/response curves for $248 \mathrm{U}$ and ara-A are very similar, and it shows that the efficacy of $1 \%$ and $2 \% 248 \mathrm{U}$ ointment, as measured by lesion inhibition, is comparable to that of $1 \%$ $\mathrm{F}_{3} \mathrm{~T}$ drops. The dose/response curve for ara-AMP indicates a substantially superior antiviral effect, but this has been shown to be due to the unique and profound lesion-inhibiting effect of ara-AMP when given shortly after inoculation (Falcon and Jones, 1977b) (Fig. 2). ara-AMP has proved too toxic for practicable clinical application to the eye.

These lesion-inhibiting experiments thus indicate a useful potential for topical $248 \mathrm{U}$, which is supported by our experiments in the treatment of established lesions (Table 1), though these have emphasised the limitations of this method.

Our experiments with intravenous $248 \mathrm{U}$ therapy demonstrate an encouraging antiviral effect in the cornea, although, as might be expected, this is delayed in comparison with the effect of topical treatment. Experiments are now in progress to establish a model of deep herpetic keratitis and to investigate the role of subconjunctival and systemic $248 \mathrm{U}$ therapy for it. These routes may prove to be the most effective means of achieving a good intraocular level of the drug, since very low aqueous levels of $248 \mathrm{U}$ were obtained after its application to the cornea, whether or not the epithelium was breached (Bauer, personal communication). The water solubility and low toxicity of $248 \mathrm{U}$ should provide no barriers to such therapy and indeed offer possibilities for safe and effective therapy of herpes virus infections throughout the body. 
We are indebted to the following: Wellcome Research Laboratories Ltd., Beckenham, for provision of $248 \mathrm{U}$; Parke-Davis \& Co., Ann Arbor, Michigan, USA, for provision of Ara A and Ara AMP; Mr R. Watkins, chief pharmacist, and staff, who prepared the antivirals; and Dr P. Collins, Wellcome Laboratories, for provision of HSV pools.

\section{References}

Bauer, D. J., Collin, P., Tucker, W. E. Jr., and Macklin, A. W. (1979). Treatment of experimental herpes simplex keratitis with acycloguanosine. British Journal of Ophthalmology, 63, 429-435.

Elion, G. B., Freeman, P. A., Fyfe, J. A., de Miranda, P., Beauchamp, L., and Schaeffer, H. J. (1977). Selectivity of action of an antiherpetic agent 9-(2-Hydroxyethoxymethyl) guanine. Proceedings of the National Academy of Sciences of the USA, 74, 5716-5720.

Falcon, M. G., and Jones, B. R. (1977a). Herpes simplex keratitis: animal models to guide the selection and optimal delivery of antiviral chemotherapy. Journal of Antimicrobial Chemotherapy, 3 (Suppl. A), 83-89.

Falcon, M. G., and Jones, B. R. (1977b). Antivirals for the therapy of herpetic eye disease. Transactions of the Ophthalmological Societies of the United Kingdom, 97, 330-332.

Jones, B. R., and Al-Hussaini, M. K. (1963). Therapeutic considerations in ocular vaccinia. Transactions of the Ophthalmological Societies of the United Kingdom, 83, 613-616.

Jones, B. R., Coster, D. J., Fison, P. N., Thompson, G. M., Cobo, L. M., and Falcon, M. G. (1979). Efficacy of acycloguanosine (Wellcome $248 \mathrm{U}$ ) against herpes simplex corneal ulcers. Lancet, 1, 243-244.

Schaeffer, H. J., de Miranda, P., Elion, G. B., Bauer, J. D. and Collins, P. (1978). 9-(2-Hydroxyethoxymethyl) guanine activity against viruses of the herpes group. Nature, 272, 583-585.

Tucker, W. E. Jr., Macklin, A. W., Szot, R. J., and Cline, D. (1978). Personal communication; presented in Abstract 64 18th Interscience Conference on Antimicrobial Agents and Chemotherapy, Atlanta, Georgia, USA, 1-4 October 1978. 